\title{
Synthesis and characterization of cellulosic fiber from red banana peduncle as reinforcement for potential applications
}

\begin{abstract}
Natural fiber-reinforced polymer composites (NRPCs) are replacing many synthetic fibers because of their cheap availability and their hygienic, ecological, biodegradable, and sustainable properties. This work involved extraction of new cellulosic fibers from red banana peduncle (RBP) plant and investigated its chemical composition, physical, structural, thermal, and tensile properties. RBP fibers (RBPFs) have high specific strength and good binding properties due to their light weight andpresence of high cellulose (72.9 wt\%), low lignin (10.01 wt\%), and wax (0.32 wt\%). X-ray diffraction (XRD) and Fourier transform infrared spectroscopy (FTIR) determined that RBPFs are rich in cellulose content with a crystallinity index $(\mathrm{CI})$ of $72.3 \%$. The density and diameter of the fibers were found to be about $0.896 \mathrm{~g} / \mathrm{cm} 3$ and $15-250 \mu \mathrm{m}$, respectively. The fiber was thermally stable up to $230^{\circ} \mathrm{C}$. Based on the results of this work, it seems that the properties of the fiber are a suitable candidate as a natural reinforcing material for the development of the biocomposite for potential applications.
\end{abstract}

Keyword: Red banana peduncle fiber; Thermal properties; Structural properties; Chemical analysis; Tensile properties 